\title{
Explicitly versus implicitly driven temporal expectations: No evidence for altered perceptual processing due to top-down modulations
}

\author{
Felix Ball ${ }^{1,2}$ - Rosa-Marie Groth ${ }^{1} \cdot$ Camila S. Agostino ${ }^{1,3} \cdot$ Emanuele Porcu $^{1} \cdot$ Toemme Noesselt $^{1,2}$
}

Published online: 24 December 2019

(C) The Psychonomic Society, Inc. 2019

\begin{abstract}
Learning the statistical regularities of environmental events is a powerful tool for enhancing performance. However, it remains unclear whether this often implicit type of behavioral facilitation can be proactively modulated by explicit knowledge about temporal regularities. Only recently, Menceloglu and colleagues (Attention, Perception \& Psychophysics, 79(1), 169-179, 2017) tested for differences between implicit versus explicit statistical learning of temporal regularities by using a within-paradigm manipulation of metacognitive temporal knowledge. The authors reported that temporal expectations were enhanced if participants had explicit knowledge about temporal regularities. Here, we attempted to replicate and extend their results, and to provide a mechanistic framework for any effects by means of computational modelling. Participants performed a letter-discrimination task, with target letters embedded in congruent or incongruent flankers. Temporal predictability was manipulated block-wise, with targets occurring more often after either a short or a long delay period. During the delay a sound was presented in half of the trials. Explicit knowledge about temporal regularities was manipulated by changing instructions: Participants received no information (implicit), information about the most likely cue-target delay (explicit), or received $100 \%$ valid cues on each trial (highly explicit). We replicated previous effects of target-flanker congruence and sound presence. However, no evidence was found for an effect of explicit knowledge on temporal expectations using Bayesian statistics. Concordantly, computational modelling suggested that explicit knowledge may only influence non-perceptual processing such as response criteria. Together, our results indicate that explicit metacognitive knowledge does not necessarily alter sensory representations or temporal expectations but rather affects response strategies.
\end{abstract}

Keywords Temporal expectations $\cdot$ Metacognition $\cdot$ Explicit vs. implicit learning $\cdot$ Computational models

Electronic supplementary material The online version of this article (https://doi.org/10.3758/s13414-019-01879-1) contains supplementary material, which is available to authorized users.

Felix Ball

Felix.Ball@ovgu.de

1 Biological Psychology, Faculty of Natural Science, Otto-von-Guericke-University, PO Box 4120, 39106 Magdeburg, Germany

2 Center for Behavioral Brain Sciences, Otto-von-Guericke-University, Magdeburg, Germany

3 ABINEP - International Graduate School, Otto-von-Guericke-University, Magdeburg, Germany

\section{Introduction}

Various organisms are able to utilize temporal information (extracted from their surroundings) to prepare appropriate reactions to relevant events at expected moments in time (Ball, Fuehrmann, Stratil, \& Noesselt, 2018a; Correa, Lupiáñez, \& Tudela, 2005; Jaramillo \& Zador, 2011; Mathewson, Fabiani, Gratton, Beck, \& Lleras, 2010; Rohenkohl, Cravo, Wyart, \& Nobre, 2012; Schroeder, Ball, \& Busch, 2018). This phenomenon has been termed temporal expectation (TE). Typically, TE is measured as performance increments (higher accuracies and lower response times (RTs)) whenever events occur at expected moments in time (see, e.g., Ball, Michels, Thiele, \& Noesselt, 2018b; Cravo, Rohenkohl, Wyart, \& Nobre, 2013; Rohenkohl et al., 2012). Over the last decades, researchers have investigated how different paradigms and different modalities affect TE and focused on identifying TErelated neural networks (for review, see: Coull, 2009; Coull, 
Cheng, \& Meck, 2011; Nobre \& Rohenkohl, 2014). At the same time, the question of how participants' knowledge about the temporal manipulation affects TE has received considerably less attention.

Knowledge about temporal regularities can be gathered in two different ways: participants can be explicitly instructed that temporal regularities are manipulated (e.g., by presenting informative cues) or they can implicitly learn about the temporal regularities if no explicit prior knowledge is provided. While explicit knowledge may involve the voluntary shift of temporal attention (top-down control; Coull \& Nobre, 1998), the extraction of regularities may rather be based on an automatic statistical learning mechanism and is often implicit (Ball et al., 2018a, 2018b; Hannula \& Greene, 2012; Henke, 2010; Turk-Browne, Scholl, Chun, \& Johnson, 2009; Turk-Browne, Scholl, Johnson, \& Chun, 2010). Given the distinction of knowing a rule beforehand versus gradually learning the rule, the question arises whether participants with explicit knowledge have an advantage in tasks that manipulate temporal expectations. Several alternative explanations present themselves of how explicit knowledge might affect TEs. First, TEs might be generated automatically by encapsulated bottom-up mechanisms - in these cases, explicit knowledge might not affect the strength of TE (Ball et al., 2018a, 2018b; Shen \& Alain, 2012; Visser, Tang, Badcock, \& Enns, 2014). Second, knowledge about likely time points of target occurrence might enhance perception and preparation for events at these time points, thereby increasing the size of the TE effect (Coull \& Nobre, 1998; Menceloglu, Grabowecky, \& Suzuki, 2017). Finally, participants might apply their knowledge in a truly optimal fashion and attend every relevant temporal position successively (for a discussion of optimal temporal strategies, see Ball, Michels, et al., 2018). For instance, in experiments with two cue-target delays (short and long), they would first attend the short temporal delay position, and if the target does not appear, they would attend the late temporal delay position. In fact, such strategy should rather reduce or even eliminate TE effects as all positions would be equally attended and temporal uncertainties - required to observe TE effects are reduced to a minimum (Ball et al., 2018b; Coull \& Nobre, 1998; Griffin, Miniussi, \& Nobre, 2001; Jaramillo \& Zador, 2011; Lange \& Röder, 2006; Nobre \& Rohenkohl, 2014; Sanders, 1975). This optimal strategy, the sequential sampling of temporal positions, should produce similar results to instances in which $100 \%$ valid temporal cues are provided. Hence, when cue validity is set to $100 \%$ compared to a less explicit instruction (e.g., "It is more likely that targets occur after a short delay") or when participants always attend each temporal delay sequentially, participants should perform equally well in all conditions (expected and unexpected). Hence, under these circumstances there should be no performance difference and no temporal expectation effect.
Menceloglu et al. (2017) recently attempted to investigate how different instruction types (no information vs. explicit information about temporal regularities) affect the build-up of temporal expectations within the same paradigm. To this end, the authors conducted a series of four experiments using a variable fore-period paradigm (i.e., manipulation of the likelihood of cue-target intervals). Fore-periods were either short or long, and fore-period probabilities were varied block-wise ( $80 \%$ short and $20 \%$ long or vice versa). Participants reported the identity of a central letter (e.g., "B") embedded between two congruent or incongruent flanker letters (e.g., "DBD" or "BBB"). In the first two experiments, participants received no information about the temporal manipulation (i.e., more short or long fore-periods; implicit manipulation). Experiment 2 was identical to Experiment 1 except that the interstimulus interval (ISI) between cue and target was filled with a sound. The authors' rationale for presenting the sound was that an auditory system has a higher temporal acuity, and thus adding a sound might facilitate the extraction of temporal regularities, thereby increasing the visual temporal expectation effect. The third and fourth experiments were again identical to Experiment 1 except that participants were told about the temporal manipulation (Exp. 3) or were reminded on each trial about the most probable fore-period within a block (Exp. 4). The authors reported that $\mathrm{TE}$ effects increased (i.e., $\mathrm{RT}_{\text {expected }}$ $<\mathrm{RT}_{\text {unexpected }}$ ) whenever participants received explicit instructions (Exps. 3 and 4) irrespective of the type of explicit information (indicating the most likely delay interval at the beginning of each block vs. at the beginning of each trial). Thus, their results appear to indicate that explicit metacognitive knowledge modulates the strength of temporal expectations (for instance, due to top-down control).

However, the results of the aforementioned study need to be interpreted with caution. To start with, it is questionable to what extent participants in Experiment 1 and 2 were aware of the temporal manipulation (i.e., had only implicit knowledge). In Experiment 1, the authors did not inquire about participants' metacognitive knowledge, while in Experiment 2 (based on a post-experimental interview that was not further elaborated on), most participants (13 out of 18) reported that they did not notice any temporal manipulation. This result is surprising given the clear difference between short and long sounds and the low difficulty of the task (as indicated by ceiling effects for accuracies). One would also expect that explicit instructions result in a decrease in response times. However, participants in Experiments 3 and 4 (explicit group) responded more slowly than in Experiment 2 (implicit group). Note that overall faster RTs in the implicit group could have masked potential TE effects due to floor effects (i.e., participants respond so fast that there is no further chance of improvement). Most importantly, overall differences in RT could have been driven by the independent samples (e.g., some participants not responding as fast as possible) or by the 
difference in physical stimulation, i.e., the presence or absence of sound presentation (Exp. 2: with sound, Exps. 3 and 4: without sound). This makes it unclear whether the observed differences in TE (larger differences for explicit group) were indeed driven by the instruction type itself, or rather by sound presence or by floor effects in the implicit group. Taken together, the pattern of results reported by Menceloglu et al. leaves several important questions unanswered and the main result - an enhancement of TE effects only in the explicit group - might rather be driven by limitations of the study's design than a demonstration of top-down influences of explicit knowledge.

Here we build upon the previous study by attempting to replicate and extend the results of Menceloglu et al. (2017) using an extended design. To this end, we tested in a series of three experiments whether explicit knowledge alters temporal expectation and, thus, the validity of the prior results. In Experiment 1, participants received no information (implicit, Menceloglu's Experiment 1) about the temporal manipulation (i.e., blocks containing more short or long cue-target). In Experiment 2, participants were instructed at the beginning of each block which foreperiod is most likely to occur (explicit, Menceloglu's Experiment 3). Note that there was no difference between the experiments with explicit instructions in Menceloglu's study so we opted for the simpler design. If these participants guide their temporal attention effectively to the most likely time points, we expect them to show a larger TE effect as compared to the implicit group. In Experiment 3, participants were told at the beginning of each block which fore-period is most likely to occur and they additionally received trial-by-trial cues (e.g., KURZ [short] or LANG [long]) indicating the length of the upcoming fore-period with $100 \%$ validity (highly explicit). Given that these participants always knew the relevant time interval, they should perform equally in all conditions (expected and unexpected) irrespective of the most likely target interval within each block (i.e., no TE effect). Note that the experimental designs of Experiments 1 and 2 were virtually identical to the designs used in Menceloglu's study. Further, we presented in ALL experiments the delay interval (i.e., cue-target interval) with or without a sound (sounds were only presented in Menceloglu's Experiment 2) to be able to assess the effects of sound presentation under all instruction types. Given the pattern of results in Menceloglu's study, we expected to be able to replicate four major findings: RTs times should be shortened (1) when a sound is presented, (2) when trials are congruent, and (3) when trials are expected, and (4) there should be no interaction of target letter congruence and temporal expectation. However, here we balanced the factor sound presentation (delays with and without sound) across instruction types, a confounding factor in the previous study, and it is thus open to debate whether TE effects are enhanced by metacognitive knowledge. If TEs are updated automatically, we expect that TE effects should not differ as a function of instruction type. However, if participants with explicit instructions only use their knowledge to attend the most likely time interval, these participants should show enhanced effects compared to the implicit and highly explicit groups. Finally, explicit instructions inform participants about the possible time intervals at which targets are presented and participants might be able to utilize their explicit knowledge optimally (i.e., sequentially attending each temporal position); such a strategy should result in a strong decrease or more likely elimination of TE effects under explicit instructions (as compared to no instruction) because prior knowledge effectively reduces temporal uncertainty of upcoming targets.

\section{Methods}

Note that the experimental design we used here was based on the design introduced by Menceloglu et al. (2017). For replication purposes, we kept the original design and only introduced extensions to the original paradigm (highly explicit instructions, sound and no sound trials for all instruction types, and a different form of trial randomization).

\section{Participants}

Sixty participants were tested in a series of three experiments (20 participants each). All participants provided written informed consent and reported to be free of neurological or psychiatric disorders and to have normal or corrected-tonormal visual acuity. For data post-processing we adopted the accuracy exclusion criteria from Menceloglu (mean \pm 3 $\mathrm{SD})$. However, as a reviewer pointed out, this kind of criterion chosen by Menceloglu et al. is not optimal for accuracies that are bounded between 0 and 1. Future studies might therefore opt to choose a more suitable exclusion criterion for nonreplication studies. In each Experiment, one participant was excluded due to low accuracy (Menceloglu et al., 2017). The demographic data of the remaining 57 participants are listed in Table 1:. This study was approved by the local ethics committee of the Otto-von-Guericke University, Magdeburg.

Table 1 Information about participants. Mean age \pm standard deviation of the 19 participants per experiment; their sex and handedness are listed for each experiment

\begin{tabular}{llll}
\hline Experiment & Age, y & Women & $\begin{array}{l}\text { Left- } \\
\text { handed }\end{array}$ \\
\hline Exp. 1 & $24.5 \pm 2.6$ & 9 & 2 \\
Exp. 2 & $25 \pm 4.7$ & 10 & 0 \\
Exp. 3 & $24.4 \pm 3.7$ & 9 & 2 \\
\hline
\end{tabular}




\section{Apparatus and stimuli}

Psychophysics Toolbox (Version 3; Brainard, 1997), Matlab 2012b (Mathworks Inc.,, Natick, MA, USA) and an LCD screen (22-in., $120 \mathrm{~Hz}$, Samsung 2233RZ) recommended for vision research (Wang \& Nikolić, 2011) were used for stimulus presentation. Headphones (Sennheiser HD 650) were used for acoustic stimulation (participants wore headphones during the course of the whole experiment including no-sound runs). Monitor resolution was set to $1,650 \times 1,080$ pixels and the refresh rate to $60 \mathrm{~Hz}$. The viewing distance was $102 \mathrm{~cm}$ (eyes to fixation point). A wireless mouse (Logitech M325) was used as the response device.

Target stimuli were the letters B or D $\left(0.7^{\circ} \times 1^{\circ}\right.$ visual angle). Targets were always presented together with two flanker letters at $0.25^{\circ}$ visual angle inter-letter spacing (center-to-center). Central targets could either be identical to or different from flankers. In Experiments 1 and 2, three asterisks served as visual cue $\left(2.6^{\circ} \times 0.7^{\circ}\right.$ visual angle, presented in the screen's center) to indicate the beginning of a trial. In Experiment 3, the words "KURZ" (short) or "LANG" (long) served as visual cue $\left(2.6^{\circ} \times 0.7^{\circ}\right.$ visual angle). Whenever no cue was present, a central fixation $\operatorname{dot}\left(0.25^{\circ}\right.$ visual angle $)$ was presented instead. Stimuli were presented on a light gray background (RGB: [204 204 204]). On each trial, a black rectangular frame was presented $\left(22.6^{\circ} \times 17^{\circ}\right.$ visual angle $)$ surrounding the cues and stimuli (see Fig. 1). Sound stimuli were presented binaurally with $4410 \mathrm{~Hz}$ at $60 \mathrm{dBA}$.

\section{Procedure}

Presentation of the three asterisks or the words "KURZ" or "LANG" (400-ms duration) marked the beginning of each trial. The cue was followed by a blank screen with a fixation dot which lasted for $400 \mathrm{~ms}$ (short delay) or $1,200 \mathrm{~ms}$ (long delay). The delay period was followed by the target stimulus (350 ms duration), which was letter B or D, either embedded among congruent (e.g., DDD) or incongruent letters (e.g., DBD). Participants were asked to report the identity of the central letter as accurately and as quickly as possible and used the index and middle finger of their dominant hand to respond. Key-finger combinations were counter-balanced across participants. After target offset, participants could still respond for $1,650 \mathrm{~ms}$. A $1,500-\mathrm{ms}$ inter-trial interval was introduced before the next trial began, during which a blank screen was shown (see Fig. 1 for exemplary trial sequences).

Temporal expectations were manipulated block-wise. In one block, short delay intervals were prevalent $(80 \%$ short vs. $20 \%$ long). In the other block, this relation was reversed. Congruent and incongruent trials, as well as target letters B

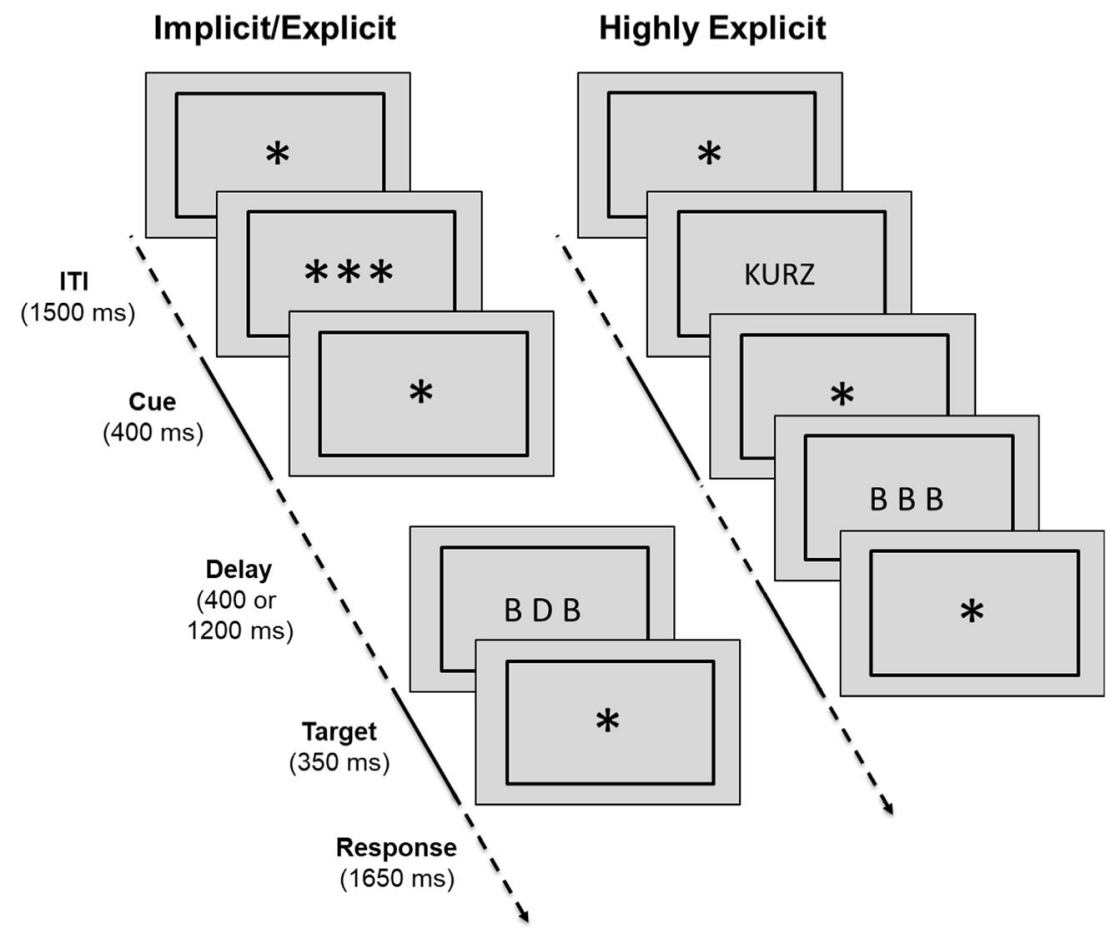

Fig. 1 Experimental design (exemplary trials). Intertrial intervals (ITIs) were set to $1,500 \mathrm{~ms}$ and followed by an uninformative cue (left sequence; implicit and explicit instructions) or an informative cue (right sequence; highly explicit instructions). After a delay of 400 or 1,200 ms (i.e., left vs. right side), a target was presented for $350 \mathrm{~ms}$ (left: incongruent, right: congruent). The response window ended 1,650 ms after target offset. Note that for the "highly explicit" instruction group, the cue (***) was substituted by $100 \%$ valid cues (KURZ [short] or LANG [long]) 
and D were balanced (50:50) within each block. Per block, we presented 200 trials in a pseudo-randomized order. In particular the same number of expected/unexpected, congruent/ incongruent and target letter (B or D) trials were presented in chunks of 20 trials. Within these chunks, trials were randomized. By equally distributing all conditions throughout the block (Ball et al., 2018a, 2018b), we assured that learning effects equally affected all conditions and that TEs could be built up continuously. As in Menceloglu's study, we labelled the first 20 trials training (i.e., the first chunk) and excluded them from further analyses.

To manipulate knowledge about temporal regularities, we altered the instructions across experiments. In Experiment 1, participants received no information about the different delay intervals and about the prevalence of short or long intervals within each block (implicit instruction type). In Experiment 2, participants were instructed about the different delays, and received information about the most likely delay interval (short or long; explicit instruction type) at the beginning of each block. In Experiment 3, we used the same instructions as in Experiment 2. However, on each trial we presented the words "KURZ" (short) or "LANG" (long) instead of the asterisks, indicating with $100 \%$ validity which delay interval was used in a given trial (highly explicit instruction type). Participants with explicit instructions were instructed to use the information provided to increase their task performance.

In addition, we presented for each participant trials with or without sound during the delay interval. The sound duration always matched the delay interval duration (400 or 1,200 ms). In each experiment, we presented four blocks - two with sound, two without sound - and the two sound or no-sound blocks were divided into one "expect early" (short delay 80\%) and one "expect late" (long delay $80 \%$ ) block. The two sound blocks (expect early, expect late) were followed by two nosound blocks or vice versa. When participants started with the "expect early" block, they did so for with and without-sound trials. The order of blocks was counterbalanced across participants. All participants (irrespective of instruction type) were told that the sound was irrelevant for the task at hand and that they could continue with the next block at their own pace.

To assess whether participants gained explicit knowledge about temporal structures in Experiment 1, we conducted a post-experiment interview (Ewolds, Bröker, de Oliveira, Raab, \& Künzell, 2017; Heuer \& Schmidtke, 1996; Nissen \& Bullemer, 1987). The following questions were always asked in an identical order to test whether participants gained knowledge throughout the experiment:

- Did you notice any regularities throughout the experiment?

- Was there something specific about the delay duration between cue and target?

- Please guess the possible delay durations.

- Was there a difference between odd and even runs?
- Was there a delay duration pattern throughout the run or was it random across trials?

- Please guess the difference between runs.

In case participants noticed the temporal regularities, we further inquired when they started noticing them.

\section{Analysis}

As in the previous report (Menceloglu et al., 2017), only early target trials were included in the analysis. Late target performance, as in Menceloglu et al., was not analyzed further as late targets are always expected and may thus not require temporal attention (Ball et al., 2018b; Coull \& Nobre, 1998; Griffin et al., 2001; Jaramillo \& Zador, 2011; Lange \& Röder, 2006; Nobre \& Rohenkohl, 2014; Sanders, 1975). Missed trials (i.e., no response; rejected number of trials across all conditions: $1.1 \pm 1.6$ ) and trials with RTs faster than $100 \mathrm{~ms}$ (rejected number of trials across all conditions: $.1 \pm .35$ ) were excluded from analyses. The same was true for the training trials (Menceloglu et al., 2017). Mean accuracies as well as mean RTs were created with Matlab 2012b (Mathworks Inc.) and analysed with separate repeated-measures ANOVAs in JASP (v. 0.9.2.0). We also used JASP to calculate the effect size $\left(\eta^{2}\right)$, conduct post hoc tests (simple main effects function), and calculate the Bayes factors (matched model effects; standard settings for priors and samples: $r$ scale fixed effects $=$ $.5, \mathrm{r}$ scale random effects $=1, \mathrm{r}$ scale covariates $=.354$, samples = "auto") in favor of the alternative hypothesis $\left(\mathrm{BF}_{\mathrm{incl}}\right)$. Note that $\mathrm{BF}_{\text {incl }}$ smaller than 1 are (more) in favor of the null hypothesis (Wagenmakers et al., 2018).

Menceloglu et al. analyzed the data of each individual experiment separately. Only the TE effect was finally tested for significant differences between experiments. Here we preferred to directly compare experiments by using a mixed ANOVA with within-subject factors sound (no, yes), TE (expected, unexpected), and congruence (congruent,

Table 2 Group mean accuracy scores. Average accuracy scores are listed as percent \pm standard deviation for all experimental conditions and experiments

\begin{tabular}{|c|c|c|c|c|c|}
\hline \multirow[t]{2}{*}{ Sound } & \multirow[t]{2}{*}{ Experiment } & \multicolumn{2}{|l|}{ Expected } & \multicolumn{2}{|l|}{ Unexpected } \\
\hline & & Congruent & Incongurent & Congruent & Incongurent \\
\hline \multirow[t]{3}{*}{ No } & 1 & $98.2 \pm 1.5$ & $97.7 \pm 2$ & $98 \pm 3.3$ & $97.7 \pm 3.4$ \\
\hline & 2 & $98.5 \pm 1.9$ & $97.9 \pm 2.6$ & $98.5 \pm 2.5$ & $96.8 \pm 4.3$ \\
\hline & 3 & $98.2 \pm 2.8$ & $97.3 \pm 3.9$ & $97.7 \pm 3.8$ & $98.5 \pm 2.5$ \\
\hline \multirow[t]{3}{*}{ Yes } & 1 & $97.7 \pm 2.8$ & $96.9 \pm 2.9$ & $98.2 \pm 3.2$ & $96.8 \pm 4.3$ \\
\hline & 2 & $98.5 \pm 1.8$ & $96.6 \pm 3.3$ & $98 \pm 2.8$ & $96.8 \pm 3.4$ \\
\hline & 3 & $98.1 \pm 2$ & $96.8 \pm 3.6$ & $97.7 \pm 2.8$ & $94.7 \pm 8$ \\
\hline
\end{tabular}


incongruent) and between-subject factor Experiment (Exps. 1-3) to be able to capture the influence of all manipulated factors.

\section{Results}

\section{Accuracy data}

Accuracy was close to ceiling in all three experiments (see Table 2). Thus, any observed effects need to be treated with caution. For completeness and comparability with Menceloglu et al., we still report the results of the statistical analysis: Performance was only affected by the sound and congruence manipulation. Accuracies increased for congruent compared to incongruent targets $(\mathrm{F}(1,54)=14.647, \mathrm{p}<.001$, $\left.\eta^{2}=.211, \mathrm{BF}_{\text {incl }}=295.054\right)$ but decreased when a sound was presented compared to the no-sound condition $(F(1,54)=$ $\left.6.146, \mathrm{p}=.016, \eta^{2}=.1, \mathrm{BF}_{\text {incl }}=2.292\right)$, though it should be noted that the mean difference across conditions was relatively small (1-2\%, see Fig. 2A and B). The Bayes factor for the interaction of Sound $*$ Congruence was in favor of neither the alternative nor the null hypothesis $(\mathrm{F}(1,54)=2.821, \mathrm{p}=.099$, $\left.\eta^{2}=.048, \mathrm{BF}_{\text {incl }}=1.181\right)$. Here, performance declined for the sound compared to the no-sound condition but only in the incongruent $(\mathrm{F}=6.04, \mathrm{p}=.017)$ and not the congruent condition $(\mathrm{F}=.17, \mathrm{p}=.682)$. All remaining effects were nonsignificant; accordingly, the BFs provided evidence for a higher probability of $\mathrm{H} 0$ (all $\mathrm{F}<=2.335, \mathrm{p}>=.106, \mathrm{BF}_{\text {incl }}$ : .428 to .064 ; see Supplement $1 \mathrm{~A}$ for a summary of all results). More importantly, these results suggest that there appears to be no direct influence of experimental instruction on performance.

\section{Response-time data}

As hypothesized, RTs decreased for congruent compared to incongruent trials $\left(\mathrm{F}(1,52)=49.624, \mathrm{p}<.001, \mathrm{\eta}^{2}=.467\right.$, $\left.\mathrm{BF}_{\text {incl }}=312.366\right)$ for the sound compared to the no-sound condition $\left(\mathrm{F}(1,52)=7.88, \mathrm{p}=.007, \mathrm{\eta}^{2}=.11, \mathrm{BF}_{\text {incl }}=\right.$ $\left.1.184 * 10^{5}\right)$ and for expected compared to unexpected trials $\left(\mathrm{F}(1,52)=11.356, \mathrm{p}=.001, \mathrm{\eta}^{2}=.169, \mathrm{BF}_{\text {incl }}=15.603\right) . \mathrm{A}$ graphic depiction of these results can be found in Fig. 2C-E. Furthermore, sound presence interacted with $\mathrm{TE}(\mathrm{F}(1,52)=$ $7.026, \mathrm{p}=.011, \eta^{2}=.112, \mathrm{BF}_{\text {incl }}=2.211$; see Fig. $\left.2 \mathrm{~F}\right)$. The TE effect (i.e., faster RT in expected trials) was only significant when a sound was presented $(\mathrm{F}=20.182, \mathrm{p}<.001)$ but not in the no-sound condition $(\mathrm{F}=.371, \mathrm{p}=.545)$. Additionally, factor Sound also interacted with factor Experiment $\left(\mathrm{F}(3,52)=4.798, \mathrm{p}=.012, \mathrm{\eta}^{2}=.134, \mathrm{BF}_{\text {incl }}=\right.$ $2.015^{*} 10^{6}$; see Fig. $2 \mathrm{G}$ ), with RTs decreasing for the sound compared to the no-sound condition but only for the experiments with explicit instructions (Exp. 2 (explicit): $F=9.47$, p
$=.006$; Exp. 3 (highly explicit): $\mathrm{F}=12.055, \mathrm{p}=.003$ ) and not for the experiment with no instructions (Exp. 1 (implicit): $\mathrm{F}=$ $.45, \mathrm{p}=.511$ ). Furthermore, there was a trend for a triple interaction of Sound * Congruence * Experiment that was not supported by the Bayes factor $(\mathrm{F}(2,54)=3.133$, $\mathrm{p}=$ $.052, \eta^{2}=.104, \mathrm{BF}_{\text {incl }}=.146$; see Fig. $\left.2 \mathrm{H}\right)$. Here, RTs decreased with sound presence in the congruent/incongruent conditions, but again, only in Experiments 2 and 3 (Exp. 1: all $\mathrm{F}<=.976, \mathrm{p}>=.336$; Exp. 2 : all $\mathrm{F}>=4.985, \mathrm{p}<=.038$; Exp. 3: all $\mathrm{F}>=11.493, \mathrm{p}<=.003)$. Most importantly, there was no evidence for an interaction of factor Experiment with factor TE (see Fig. 2I for a graphic depiction of Sound * TE * Experiment) and all remaining effects were neither significant nor supported by the Bayes factor (all $\mathrm{F}<=1.359, \mathrm{p}>=.266$, $\mathrm{BF}_{\text {incl }}: .331$ to .078 ; see Supplement $1 \mathrm{~B}$ for a summary of all results).

\section{Re-analysis based on subjective reports 1: All experiments}

So far, we have only reported effects that included the analysis of all participants, irrespective of their subjective reports (post-experimental interview; see detailed questionnaire results in Supplement 2).

In Experiment 1, seven out of 19 participants noticed the temporal manipulation when no sound was present. Six of them noticed the manipulation directly in the first block. When a sound was presented, 15 out of 19 participants noticed the temporal manipulation; seven of them already in the first block. Hence, our data does not match the data from Menceloglu and colleagues, as they reported that most of the participants did not notice the different sound durations and, thus, the temporal manipulation. Based on the postexperimental interview, participants in Experiment 1 can be split into three groups: those who never noticed the temporal manipulation (implicit, $\mathrm{n}=4$ ), those who only noticed it for the sound but not the no-sound condition (switch, $\mathrm{n}=8$ ) and those who noticed the temporal manipulation in both conditions (explicit [learned], $\mathrm{n}=7$ ). Based on this group split we re-ran our analysis to account for possible influences of explicit knowledge in Experiment 1. Again, we used a mixed ANOVA with within-subject factors sound (no, yes), TE (expected, unexpected), and congruence (congruent, incongruent), but this time the between-subject factor was based on the knowledge group (implicit, switch, explicit [learned], explicit, highly explicit).

Note that the results of the re-analysis were virtually identical to the results of our initial analysis. Again, accuracies increased for congruent compared to incongruent targets $\left(\mathrm{F}(1,52)=7.675, \mathrm{p}=.008, \mathrm{\eta}^{2}=.126, \mathrm{BF}_{\text {incl }}=305.478\right)$ and decreased with sound presence $\left(\mathrm{F}(1,52)=4.773, \mathrm{p}=.033, \mathrm{\eta}^{2}\right.$ $=.079, \mathrm{BF}_{\text {incl }}=2.312$, see Fig. $2 \mathrm{~A}-\mathrm{B}$ ), while all remaining effects were non-significant (all $\mathrm{F}<=1.538, \mathrm{p}>=.205, \mathrm{BF}_{\text {incl }}$ : 

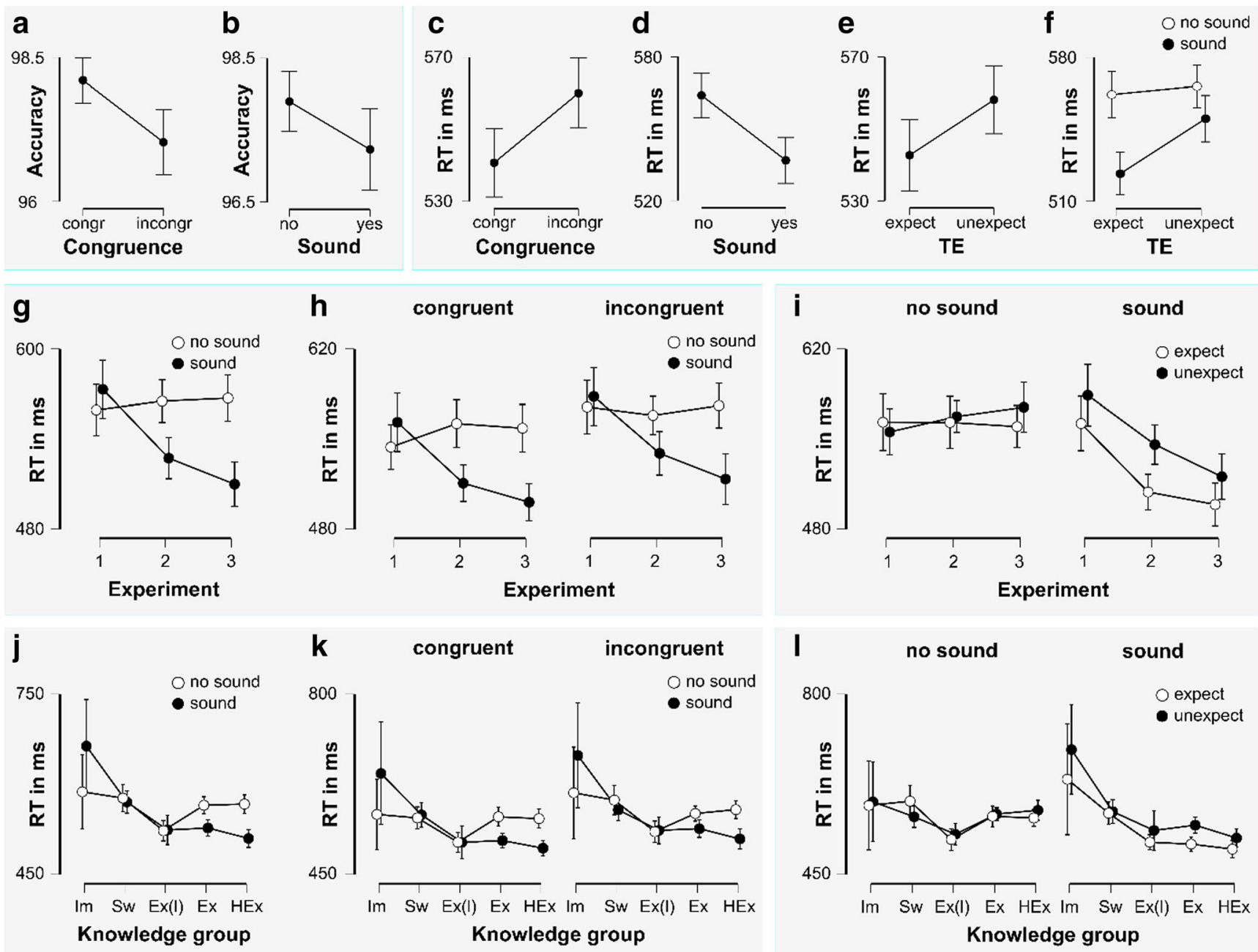

Fig. 2 Mean accuracy and reaction time (RT) results ( \pm conditionspecific confidence interval (CI)). Please note that in I and L the black/ white color code depicts different experimental factors (expected/unexpected instead of sound/no sound). (A and B) Group mean accuracy results for the congruence and sound main effects. $(\mathbf{C}-\mathbf{F})$ Group mean RT results for all effects excluding factors Experiment/Knowledge group: main effects of congruence, sound, and temporal expectation and interaction Sound * TE. (G and H) RT results for the interaction Experiment * Sound and Experiment $*$ Sound $*$ Congruence. (I) Mean RT differences between expected and unexpected trials for the two sound conditions and each experiment. ( $\mathbf{J}-\mathbf{L})$ Same as G-I but with factor Knowledge group instead of Experiment. Error bars are 95\% CIs as computed in JASP. Knowledge group: Exp. $1-I m=$ implicit, $S w=$ switch (participants unaware of temporal manipulation in no sound condition but not in sound condition), $\operatorname{Ex}(l)=$ explicit (learned; participants who became aware of the temporal manipulation in all conditions); Exp. $2-E x=$ explicit instruction; Exp. $3-H e x=$ highly explicit instruction

$.111, \mathrm{BF}_{\text {incl }}=2.483$; see Fig. $\left.2 \mathrm{~F}\right)$ and Sound $*$ Knowledge group (before Sound $*$ Experiment; $F(4,52)=3.456, p=.014$, $\eta^{2}=.209, \mathrm{BF}_{\text {incl }}=1.47 * 10^{8}$; see Fig. $2 \mathrm{~J}$ ) were again significant, with the same underlying effects: (1) The TE effect was only significant in the sound condition (no-sound: $\mathrm{F}=.028, \mathrm{p}$ $=.867$; sound: $\mathrm{F}=16.583, \mathrm{p}<.001)$ and (2) RTs decreased when a sound was present (compared to no difference in the no-sound condition), but only in Experiments 2 and 3 (Exp. 1 (all knowledge groups): all $\mathrm{F}<.776$, all $\mathrm{p}>.433$; Exp. 2 (explicit): $\mathrm{F}=9.47, \mathrm{p}=.006$; Exp. 3 (highly explicit): $\mathrm{F}=$ $12.055, \mathrm{p}=.003)$. In line with the first analysis, the interaction of Sound * Congruence * Experiment approached significance $\left(\mathrm{F}(4,52)=2.201, \mathrm{p}=.082, \eta^{2}=.144, \mathrm{BF}_{\text {incl }}=.09\right.$; see Fig. 2K), with faster RTs with sound presence in both the interactions of Sound $*$ TE $\left(F(1,52)=6.854, p=.012, \eta^{2}=\right.$ 
congruent and the incongruent conditions of Experiments 2 and 3 (Exp. 1 (all knowledge groups): all $\mathrm{F}<=1.066, \mathrm{p}>=$ .348; Exp. 2 (explicit): all $\mathrm{F}>=4.985, \mathrm{p}<=.038$; Exp. 3 (highly explicit): all $\mathrm{F}>=11.493, \mathrm{p}<=.003$ ). Most importantly, accounting for explicit knowledge in Experiment 1 did not result in statistically significant interactions of factor Knowledge group with factor TE (see Fig. 2L for a graphic depiction of Sound $* \mathrm{TE} *$ Knowledge group) as all remaining effects were again neither significant nor supported by the Bayes factor (all $\mathrm{F}<=1.945, \mathrm{p}>=.117, \mathrm{BF}_{\text {incl }}: .381$ to .033 ; see Supplement $3 \mathrm{~B}$ for a summary of all results).

\section{Re-analysis based on subjective reports 2: Individual conditions/experiments}

Note that splitting participants into the different Knowledge groups came at the cost of lower sample sizes for each Knowledge group in Experiment 1, thereby increasing the standard deviation of the mean, especially in the implicit group (see Fig. 2J-L). To further support the notion that the large-scale ANOVA does not conceal potential differences between knowledge groups, we conducted two complementary analyses. First, we repeated the analysis, but separately for the sound/no-sound conditions (with factors TE, Congruence, and Knowledge group; see Supplement 4). By removing the sound factor, there was no need to classify participants in Experiment 1 as "switcher," and we only used the implicit and explicit [learned] labels, resulting in a four-level factor of Knowledge group (implicit, explicit [learned], explicit, and highly explicit). This automatically increased the sample size of the implicit group in Experiment 1 in the no-sound condition $(n=12)$, which was the crucial condition in Menceloglu et al.'s experiment (three out of four experiments had no sound presentation).

We concur, however, that this analysis does not necessarily solve the issue of the low implicit group sample size $(n=4)$ in the sound condition. However, given our post-experimental reports, we would need to collect data from at least 100 participants to have a sufficient sample size of approximately 20 implicit participants in the sound condition. Further, it is questionable to which extent the reported results would change: Ranking the TE effects by their magnitude (max. to min.) in the sound condition, the four implicit participants would have the ranks 1, 12, 37, and 49 (out of all 57 participants). The TE effect of the two best ranked participants exceeded the TE effects of 16 (out of 19) participants in Experiment 2 and 15 participants in Experiment 3. Even the third best participant in the implicit group had a larger TE effect than ten participants in Experiments 2 and 3 (three participants in Exp. 2, seven participants in Exp. 3). Thus, our data do not indicate overall smaller TE effects in the implicit group compared to the explicitly instructed groups. However, given the small sample size $(n=4)$ in the sound condition, this inevitably increased the confidence interval shown in Fig. 2J-L (hence, these intervals cannot be taken as an indication of higher temporal precision in the explicit groups).

Nonetheless, the aforementioned reasoning does not fully address the problem of the low sample size in the sound condition only. On a reviewer's suggestion, we took into account the time point when participants became aware of the temporal manipulation in the sound condition. Eight participants became aware of the temporal manipulation during the second block of the sound condition. The results of these participants could thus be interpreted as "mainly implicit." Together with the four truly implicit participants, the sample size would be increased to 12 for the implicit group. We ran an additional ANOVA only testing for differences in temporal expectation (expected, unexpected) and by only taking into account three groups (implicit, explicit [Exp. 2], and highly explicit [Exp. $3]$ ), thereby increasing the power to potentially detect differences. However, this analysis also revealed no difference between groups (interaction of TE $*$ group: $\mathrm{F}(1,47)=.876, \mathrm{p}=$ $\left..423, \mathrm{BF}_{\text {incl }}=.29\right)$. Even if we limit the implicit participants to only those who became aware of the temporal manipulation for the unexpected trials $(n=8$; thereby potentially decreasing the difference between expected and unexpected trials), the result did not change (interaction of $\mathrm{TE} *$ group: $\mathrm{F}(1,43)=$ $.754, \mathrm{p}=.477, \mathrm{BF}_{\text {incl }}=.295$ ).

In addition to the abovementioned analysis, we also analysed the data separately for each experiment and the sound and no-sound conditions (repeated-measures ANOVA with factors $\mathrm{TE} *$ Congruence [plus Knowledge group in Experiment 1]). This analysis is closest to the one reported in Menceloglu et al. (2017). The results of this analysis, including graphs for significant effects, are listed in Supplement 5. Importantly, note that the results of both additional analyses are in line with the main results reported in this manuscript and suggest that TE effects were only present in the no-sound condition, but more importantly, for all instruction types.

\section{Discussion}

In this study, we investigated whether temporal expectation (TE) differentially affected performance regarding temporally expected and unexpected targets. The critical comparison contrasted participants with implicit versus explicit knowledge about temporal regularities, in an attempt to replicate and extend a recent study (Menceloglu et al., 2017). To this end, we used an identical paradigm but extended it by (1) introducing a new form of explicit instructions in Experiment 3 (i.e., $100 \%$ valid feedback about the upcoming delay interval between cue and target), by (2) adding the factor sound (present/absent) in the cue-target interval for all three instruction types (no information [implicit], explicit, highly explicit) and by (3) analyzing the data in accord with 
participants' metacognitive status. Most importantly, by keeping the physical stimulation (sound/no sound) identical across experiments, we were able to distinguish between the influence of instruction type and, thus, participants' metacognitive knowledge about temporal regularities versus the influence of sound presence. As hypothesized, we were able to replicate four effects of Menceloglu's study. Participants responded faster when trials were expected, when targets were congruent with flankers, and when the cue-target interval was filled with an irrelevant sound. As in the previous paper, we also found no evidence for an interaction of congruence * temporal expectation. Remarkably, while most of our results are in good agreement with the earlier study, the critical comparison did not support the conclusion that explicit knowledge generally enhances temporal expectation effects. This suggests that explicit knowledge may not always affect temporal expectations.

\section{Comparison with the previous study}

While we tried to improve on the original design by Menceloglu et al. (2017), there was one factor - explicit knowledge - that could not be manipulated within participants. As in the previous study, we recruited different participants for the individual experiments. Note that if the same participants are tested under all instruction types, participants who first receive explicit instructions are not naïve when continuing with the no-instruction experiment. Hence, these participants could never be labelled "implicit." In turn, if participants always start with the no-instruction experiment, ordering effects may occur, for example, participants would always be more trained under explicit instructions. To avoid ordering effects, we chose to collect data from independent samples.

There are several alternative explanations for the replication failure of the TE effects described by Menceloglu et al. (2017). In general, we found only small improvements due to temporal expectations in the present study, with an average improvement (across all experiments) of $16 \mathrm{~ms}$ for expected compared to unexpected trials. This finding might be attributed to the low task difficulty in the present experiment, as indicated by fast RTs and low error rates (in contrast to other TE studies with more complex tasks; see, e.g., Ball et al., 2018a, 2018b): participants responded on average within 500-600 ms across all conditions and instruction types, hence, overall faster than in Menceloglu et al.'s study. Thus, the average difference in RTs between groups in our study was minimized, rendering the TE effects across groups/experiments more comparable. To recap, in Menceloglu's study, average RTs between 560 and $740 \mathrm{~ms}$ depending on the individual experiment, with overall slower average RTs $(600-740 \mathrm{~ms})$ in their experiments with explicit instructions and no sound presentation (Exps. 3 and 4) compared to their experiment with no instructions and sound presentation (Exp. 2; RTs of 560-640 ms). At the same time, the TE effect was larger in their experiment with explicit instructions (no sound) compared to their Experiment with no instructions (sound). Thereby, differences in TE effects between instruction types in the earlier study might have been driven by differences in average response speed, a factor that was not taken into account. Further, it remains unclear whether the physical stimulation itself (sound vs. no sound) or participants' knowledge about temporal regularities were driving the differences in TE effects in the prior study as the sound appears to have altered the response criterion (participants responded approx. $100 \mathrm{~ms}$ faster in the sound condition). Here we have shown that when participants adhere to the general instructions and respond as quickly and accurately as possible, this response criterion appears to eliminate differences in performance between instruction types.

Additionally and most importantly, we were not able to replicate the finding that - without presenting a sound - participants with explicit instructions show a reliable temporal expectation effect (i.e., RT expected faster unexpected; Exp. 2 in the present study, Exps. 3 and 4 in the previous study). In our Experiment 2, the average difference between expected and unexpected trials was as small as $4.5 \mathrm{~ms}$ compared to approximately $60 \mathrm{~ms}$ in the previous study. As mentioned, the different magnitudes of TE effects across studies could be partially driven by the difference in average response speed across studies. However, our data also suggest that there is no mandatory relationship between response speed and the presence and size of TE effects. In particular, TE effects were only significant when a sound was presented, the very condition in which participants in our Experiments 2 and 3 responded fastest. Hence, faster responses do not eliminate or minimize the possibility of the presence of TE effects. Furthermore, participants in the implicit group showed larger TE effects in the sound condition although they responded on average slower than all other subjects in the sound condition. But correlating the average RT and the size of TE effect across all participants did not reveal a direct relation of both factors. Thus, average response speed alone cannot easily account for the difference in results.

In our study, TE effects - under all instruction types - were only significant when a sound was present. Given that we presented the sound as well as the no-sound condition within the same session leaves the possibility that the TE effect was dependent on whether participants started with the sound presentation. Starting with the sound presentation (the condition that provided more information on the delay interval lengths) might have decreased TE effects in the no-sound condition because participants were confronted with a more difficult set up in the second half of the experiment (no direct temporal information). This might have discouraged them to adhere to the task instructions. However, we found no evidence for such an order effect (i.e., sound first or second; see Supplement 6). Ruling out an order effect of the sound presentation, our 
results could potentially imply that temporal expectations are simply less stable or less coherently updated/created without the additional sound presentation when easy task regimes are used. In turn, the behavioral outcome (i.e., the presence of a TE effect) in the absence of sound presentation would vary depending on the sample group but irrespective of the instruction type. In principle, the null finding in the no-sound condition appears to be odd given that there are a variety of visual studies indicating that RTs are typically facilitated in expected trials (for a review, see: Coull, 2009; Coull et al., 2011; Nobre $\&$ Rohenkohl, 2014). However, we were able to replicate Menceloglu et al.'s null finding of their Experiment 1 (no instructions and no sound). Further, we previously showed that only multisensory targets resulted in robust temporal expectations as compared to unimodal auditory and visual targets (Ball et al., 2018a, 2018b). Especially in the visual condition, effects seemed to be less pronounced in our experiments. Thus, yet another possibility is that the absence of unimodal (here visual) TE effects might be simply underrepresented in the literature due to publication bias favoring positive results when only unimodal conditions are tested.

It is also worth mentioning that we were also not able to replicate Menceloglu et al.'s post-experimental debriefing results. After presenting a sound in the cue-target interval, almost all participants (16 out of 20 ) receiving no instructions about temporal regularities reported noticing the difference of short and long sounds, and, thus, the difference of short- and long-delay intervals. This is in clear contrast to Menceloglu et al.'s study in which it was reported that most participants under the same condition - did not notice this difference. The post-experimental debriefing relies on participants' understanding of the questions they are being asked. In our experience, not all participants fully understand what is meant by the question "Did you notice any regularities throughout the experiment?" This open question has to be accompanied by further questions allowing participants to guess whether or not temporal regularities were manipulated. By using a more exhaustive post-experimental questionnaire, we were able to identify that almost all participants noticed the manipulation of temporal regularities when a sound was presented. In the study by Menceloglu and colleagues, participants in the implicit group were asked whether they noticed differences in trial timing or tone duration (1) across blocks or (2) within each block. If they responded positively, the authors asked them to further elaborate to assess the relevance of the answer. Hence, these questions are similar to the ones we asked. Either participants in the earlier study were truly unaware of the differences in sound duration (which renders the difference across studies a sample effect) or they did not fully understand the questions (hence, some implicit participants might have had a mild form of explicit temporal attention). To be able to draw a stronger conclusion, future studies are required to assess how the individual sample, the phrasing of questions, and task difficulty affect participants' statements about their metacognitive knowledge of temporal manipulations.

\section{The validity of temporal expectation effects: Bottom-up, top-down or bias?}

Irrespective of which factor was causing the differences between our and the previous study, our results mainly indicate that metacognitive knowledge in the present paradigm does not enhance temporal expectation effects. Following up on our initial hypotheses of how explicit knowledge might affect TE effects, our results do not favor the suggestion that (1) explicit knowledge enhances TE effects and that (2) highly explicit knowledge eliminates TE effects. Thus, for all types of explicit knowledge, participants rather tended to take into account the general likelihood of early and late trials within a block without any improvement as compared to the implicit group. While our results were based on the average across all trials, two reviewers suggested that effects between explicit and implicit participants might be more pronounced at the start of each block (i.e., when participants with implicit knowledge first have to learn the temporal rules; i.e., block with more short or long delays). To this end, we added another analysis and calculated average RTs for the expected/unexpected and sound/no sound conditions for the first 20,40,60, 80, and 100 trials (five individual ANOVAs; see Supplement 7). Note that the first 20 trials were the training trials that were discarded for the other analyses. However, even when taking into account only the first trials of each block (instead of averaging all trials), we found no difference between the individual groups' TE effects.

This suggests that temporal expectation effects in the current study might have been based on automatic processing of temporal regularities. This suggestion would be in line with a variety of studies demonstrating participants' ability to implicitly improve performance due to statistical (Hannula \& Greene, 2012; Henke, 2010; Turk-Browne et al., 2009, 2010) and perceptual learning (Seitz, 2017; Seitz \& Watanabe, 2009); in addition, studies on temporal attention showed that target detection in attentional blink paradigms and block-wise manipulations of TEs can be based on implicit learning (Ball et al., 2018a, 2018b; Shen \& Alain, 2012; Visser, Ohan, \& Enns, 2015). Our results are also in line with results from various other paradigms - such as contextual cueing, perceptual priming, motor sequence learning, and timing tasks - showing no improvement in behavioral effects when comparing influences of implicit and explicit knowledge on performance within the same paradigm (Chun \& Jiang, 2003; Francken, Gaal, \& de Lange, 2011; Geyer, Baumgartner, Müller, \& Pollmann, 2012; Max, Widmann, Schröger, \& Sussman, 2015; Preston \& Gabrieli, 2008; Sanchez \& Reber, 2013; Van den Bussche et al., 2013; Westerberg, Miller, Reber, Cohen, \& Paller, 2011). 
However, there is also evidence that for highly deterministic sequences, explicit instructions might decrease RTs (Stefaniak, Willems, Adam, \& Meulemans, 2008), that explicit instructions can generally improve performance at the cost of reducing condition-specific benefits (Schlagbauer, Muller, Zehetleitner, \& Geyer, 2012), or that they result in a change of response strategy (Schlagbauer et al., 2012; Summerfield \& Egner, 2009). Thus, whether or not TE effects are enhanced by top-down control might be based on the need for performance improvement depending on the task regime.

Complementary analysis: Drift-diffusion modelling approach

Our results (see also Supplemental Material for further corroborating results) did not provide direct support for a difference between instruction types, leaving the possibility that temporal expectations are always created automatically. However, it might be that participants with explicit knowledge adopted a more constant and liberal response criterion and responded overall faster in the expected condition at the cost of more errors (Schlagbauer et al., 2012; Summerfield \& Egner, 2009). In fact, one reviewer suggested transforming the accuracy scores (arcsin), as accuracies were close to ceiling, to avoid misinformed conclusions based on the ANOVA results. These arcsin-based results (Supplement 8) suggest that participants generally performed lower in the expected condition, which - together with the RT patterns - suggests a speedaccuracy trade-off.

To provide a more mechanistic explanation of our results and to test the hypothesis that the effects presented here resemble a speed-accuracy trade-off rather than true perceptual facilitation, we added a complementary analysis and computed a set of drift-diffusion models (DDMs) (Jepma, Wagenmakers, \& Nieuwenhuis, 2012; R Ratcliff, 1979; Roger Ratcliff \& McKoon, 2008; Roger Ratcliff \& Rouder, 1998). DDMs assume that stimulus information is noisy and evidence about a stimulus is accumulated over time. The decision process is modelled by three main parameters (see Fig. 3A): non-decision time ( $\mathrm{T}_{\mathrm{er}}$; combines stimulus encoding and speed of response execution), boundary separation (a; criterion to initiate a response), and drift rate ( $\nu$; mean rate of evidence accumulation). The DDMs were computed with the DMAT toolbox for Matlab (version 0.4; Joachim Vandekerckhove \& Tuerlinckx, 2008; Joachm Vandekerckhove \& Tuerlinckx, 2007) and models were specified in close resemblance to the DDM settings used by Jepma et al. (2012), who investigated how word recognition is affected by short and long cue-target fore-periods. We computed the DDMs separately for each experiment with the following base conditions: (1) the starting point of the diffusion process ( $\mathrm{z}$ ) was free to vary between the congruent and incongruent condition while the variability of the starting point (sz) was kept constant across conditions. (2) The mean drift rate and the variability of the drift rate $(\eta)$ were allowed to vary between the congruence conditions. (3) The inter-trial variability of the non-decision time (st) was allowed to vary between sound conditions (Models 1-5), expected and unexpected trials (Models 6-10), or between all sound and TE conditions (Models 11-15). (4) Models 1, 6, and 11 were computed with fixed boundary separation (a), non-decision time (Ter) and drift-rate $(v)$ across conditions. In all other models we allowed either boundary separation (a), non-decision time (Ter), driftrate $(v)$, or all three parameters to vary. In Models $1-5$ parameters varied for sound only, in Models 6-10 for TE only, and in Models 11-15 for sound * TE. The best fitting models were identified by means of the Bayesian information criterion (BIC; see Fig. 3B). Furthermore, we used the average group data to fit the model as there were only few error responses on the single subject level (Jepma et al., 2012; R Ratcliff, 1979; Roger Ratcliff \& McKoon, 2008; Roger Ratcliff \& Rouder, 1998). A summary of the models as well as plots for all Experiments can be found in the Supplementary Material (Supplement 9). An exemplary plot of the model fit for Experiment 1 can be found in Fig. 3C. Please note that by means of DDMs, we were able to test which underlying mechanism (change of response preparation, evidence accumulation or a shift in response bias) best explains the data. If our data are mostly based on a speed-accuracy trade-off, we should find that the best model (in the explicit or even all groups) contains a shift of the response bias parameter rather than changes in the non-decision time or drift-rate. Given the rather small effects of TE in this study, the DDMs also help to disentangle to which degree temporal expectations were relevant for task performance in the individual groups. If temporal expectation enhanced performance and operates similar in the present paradigm as compared to previous studies, the best fitting model should incorporate the manipulation of TE and an alteration of either the non-decision time and/or drift rate.

The results of the DDM analyses suggest that TE had little influence in general in the current paradigm (which is in line with the small average improvement of the group mean RTs by TE). More importantly, the results for Experiments 2 and 3 even suggest that explicit knowledge rather changes the response criterion in form of a speed-accuracy trade-off. For Experiment 2, the best model was a combination of the influence of sound and TE. However, only the boundary separation was affected by these two parameters with overall lower boundaries in the sound compared to no sound condition, and also lower boundaries in the expected versus unexpected condition (no sound: expected $=.165$, unexpected $=.174$; sound: expected $=.144$, unexpected .161). As can be seen from the numbers, the change in boundary between expected and unexpected trials was also almost twice as high in the sound condition. In Experiment 3, TE had no effect on the data but only the sound. The best model suggests that the sound changed the boundary, non-decision time as well as the drift. However, while the drift rate was higher in the sound 
a

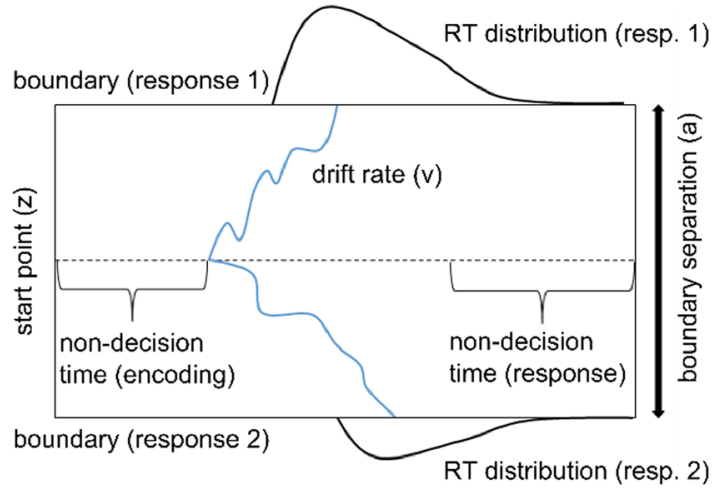

b

$$
\text { b }
$$
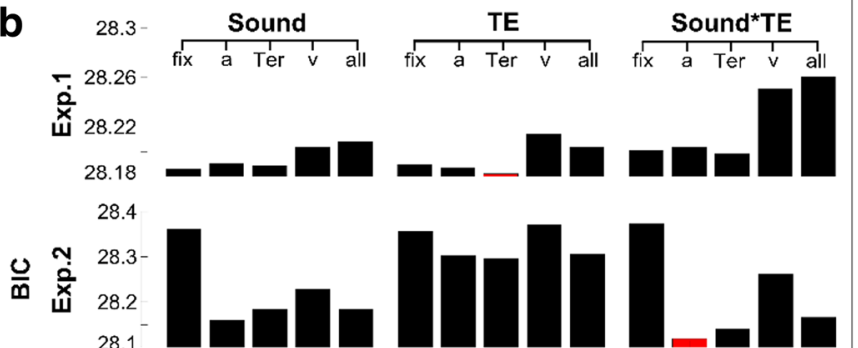

28.7

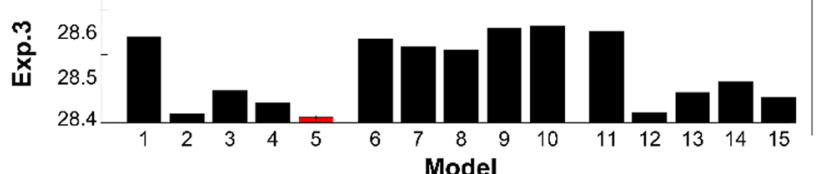

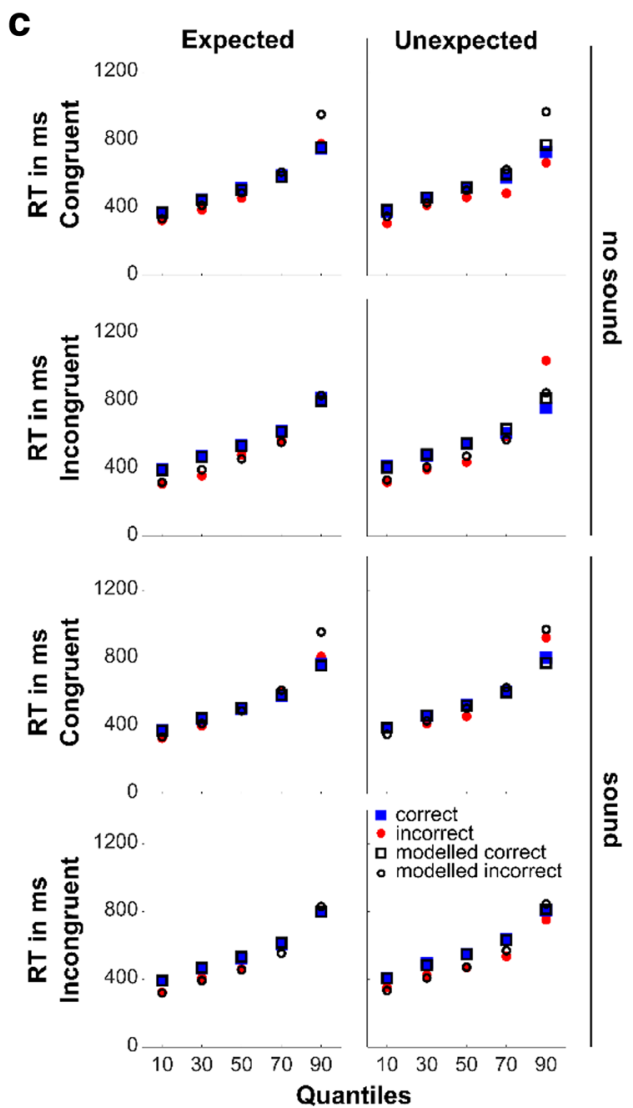

Fig. 3 Drift diffusion model analysis: (A) Graphic depiction of drift diffusion model with individual parameters: boundary separation (a), non-decision time $\left(\mathrm{T}_{\mathrm{er}}\right)$, drift rate $(\mathrm{v})$, starting point $(\mathrm{z})$. (B) Bayesian Information Criterion (BIC, arbitrary units) for all models and all Experiments (Exps. 1-3 from top to bottom). The top row header indicates which parameters of the model were free to vary (fix $=$ none, all $=a$, Ter and v) for which condition (sound, TE, or Sound \& TE). The best model (lowest BIC) for each Experiment is highlighted in red. An

compared to the no-sound condition (no sound $=.4$, sound $=$ .45 ), the opposite was true for the boundary separation (no sound $=.166$, sound $=.133$ ). This pattern again provides evidence for a speed-accuracy trade-off as typically an increase of both, boundary and drift rate is found in tasks with accuracy emphasis (Zhang \& Rowe, 2014). Finally, TE affected the non-decision time in Experiment 1 with slightly faster nondecision times in the expected condition (expected $=.277$, unexpected $=.292$ ). This result is in line with previous studies manipulating TE and using only implicit instructions (Jepma et al., 2012) and it might indicate a shift in response preparation on expected trials (for a discussion of changes in Ter resembling changes in encoding or response preparation, see Jepma et al., 2012). Nevertheless, we would like to emphasize that the second best model for Experiment 1 was one of the base models not including changes of boundary, non-decision time, and drift rate.

In sum, the results of the DDM suggest that explicit instructions change participants' response criterion at the cost of producing more errors which was not covered by the exhaustive description of individual models (with varying parameters) can be found in Supplement 9. (C) Exemplary result of the model fit. Here we show the data for Experiment 1 (see Supplement 9 for remaining fits). The plots depict the comparison of original reaction time (RT) data quantiles (red circles - incorrect responses, blue squares - correct responses) and modelled RT data quantiles (black circles/black squares). RT data are presented in milliseconds. The better the model fit, the more filled and empty markers overlap

Frequentist and Bayesian statistical analysis. However, note that - at least on average - participants with explicit knowledge showed reduced accuracy compared to participants with implicit knowledge and an overall accuracy decrease in the sound condition (see Supplement 9) while at the same time making faster responses. This pattern is indeed indicative of a speed-accuracy trade-off. Given that the two explicit groups show a similar pattern (namely indications for a speed-accuracy-trade-off), although being independent of one another, while the implicit group does not, indicates that the instruction itself influenced the outcome of the DDM rather than the individual participants.

Please note that we added the DDM as well as the analyses suggested by reviewers in the Discussion, as these analyses were not pre-planned but they provide additional insights into the origin of effects presented here and in earlier studies. Moreover, in the current paradigm there were only few erroneous responses and performance was almost at ceiling - as our aim was to replicate a previous study, not to use a design optimized for DDMs. Thus, additional studies are required 
with designs optimized for computational modelling (e.g., a larger number of trials and a more complex task to obtain a higher number of erroneous responses) to further support the current findings.

\section{Bottom-up, top-down or bias?}

The results presented here suggest that although the average RT effects appeared to be qualitatively similar, they are potentially based on different mechanisms as indicated by the DDM. Thus, similar appearing average behavioral readouts across groups might be driven by different neural processes (see, e.g., Max et al., 2015; Preston \& Gabrieli, 2008). Putting the DDM results in context with our hypothesis, they suggest that the temporal expectation effects were driven not only by bottom-up but also top-down effects. However, the results also question what the TE effects in this and also the prior study actually represent. Our results indicate that performance in the expected compared to the unexpected condition do not necessarily imply (1) higher response preparation and (2) facilitated stimulus processing, two factors which are typically suggested to explain temporal expectation effects. Depending on the task and in the absence of a clear improvement of accuracies, average RT effects are hardly interpretable and TE effects might rather imply changes in response strategies but not perceptual sensitivity per se (Schlagbauer et al., 2012; Summerfield \& Egner, 2009). In these cases, future studies should incorporate - if possible - other analyses such as computational modelling to be able to make more informed decisions about the origin of effects.

\section{Conclusion}

To summarize, here we opted for an established paradigm that has been previously used to investigate the effects of implicit and explicit temporal knowledge on temporal expectation effects. A previous report suggested that explicit knowledge increases the benefits of TE; however, this result was not purely based on differences in explicit vs. implicit knowledge but also on differences in physical stimulation, i.e., the presence and absence of sound stimulation. Here we investigated implicit and explicit temporal expectation effects - both in the presence and the absence of sounds. To start with, we were not able to replicate the presence of TE effects in the absence of sound stimulation. Most importantly, our results suggest that explicit metacognitive knowledge does not enhance temporal expectation compared to implicit knowledge. Rather, it appears that especially explicit knowledge led to a change in response criteria, with more liberal responses in the explicit instruction groups. In sum, our findings strongly suggest that researchers have to be careful when interpreting TE effects that are solely based on average RTs and that learning and utilization of statistical temporal information is not always enhanced by explicit knowledge.

Acknowledgements This work was funded by the European Fonds for regional Development (EFRE), ZS/2016/04/78113, Center for Behavioral Brain Sciences - CBBS. We thank three anonymous referees for their helpful comments.

Author contributions statement F.B. designed the experiment. R.M.G acquired the data. F.B. analysed the data. All authors wrote and revised the manuscript.

\section{Open Practices Statement}

Code availability Data were analysed with JASP (freely available) and the DMAT toolbox for Matlab (version 0.4). The remaining code is available upon request.

Data availability The authors declare that the analysed data as well as the supplementary analyses (not reported in the manuscript) are available in the Supplementary Information files.

\section{Compliance with ethical standards}

Competing interests The authors declare no competing financial and non-financial interests, or other interests that might be perceived to influence the results and/or discussion reported in this paper.

\section{References}

Ball, F., Fuehrmann, F., Stratil, F., \& Noesselt, T. (2018a). Phasic and sustained interactions of multisensory interplay and temporal expectation. Nature Scientific Reports, 8, 10208. https://doi.org/10.1038/ s41598-018-28495-7

Ball, F., Michels, L. E., Thiele, C., \& Noesselt, T. (2018b). The role of multisensory interplay in enabling temporal expectations. Cognition, 170, 130-146. https://doi.org/10.1016/j.cognition.2017. 09.015

Brainard, D. H. (1997). The Psychophysics Toolbox. Spatial Vision, 10(4), 433-436. https://doi.org/10.1163/156856897X00357

Chun, M. M., \& Jiang, Y. (2003). Implicit, long-term spatial contextual memory. Journal of Experimental Psychology. Learning, Memory, and Cognition, 29(2), 224-234. Retrieved from http://www.ncbi. nlm.nih.gov/pubmed/12696811

Correa, Á., Lupiáñez, J., \& Tudela, P. (2005). Attentional preparation based on temporal expectancy modulates processing at the perceptual level. Psychonomic Bulletin \& Review, 12(2), 328-334. Retrieved from http://www.ncbi.nlm.nih.gov/pubmed/16082814

Coull, J. T. (2009). Neural substrates of mounting temporal expectation. PLoS Biology, 7(8), e1000166. https://doi.org/10.1371/journal.pbio. 1000166

Coull, J. T., Cheng, R.-K., \& Meck, W. H. (2011). Neuroanatomical and Neurochemical Substrates of Timing. Neuropsychopharmacology, 36(1), 3-25. https://doi.org/10.1038/npp.2010.113

Coull, J. T., \& Nobre, A. C. (1998). Where and when to pay attention: the neural systems for directing attention to spatial locations and to time intervals as revealed by both PET and fMRI. Journal of Neuroscience, 18(18), 7426-7435. https://doi.org/0270-6474/98/ $187426-10 \$ 05.00 / 0$

Cravo, A. M., Rohenkohl, G., Wyart, V., \& Nobre, A. C. (2013). Temporal Expectation Enhances Contrast Sensitivity by Phase Entrainment of Low-Frequency Oscillations in Visual Cortex. 
Journal of Neuroscience, 33(9), 4002-4010. https://doi.org/10. 1523/JNEUROSCI.4675-12.2013

Ewolds, H. E., Bröker, L., de Oliveira, R. F., Raab, M., \& Künzell, S. (2017). Implicit and Explicit Knowledge Both Improve Dual Task Performance in a Continuous Pursuit Tracking Task. Frontiers in Psychology, 8, 2241. https://doi.org/10.3389/fpsyg.2017.02241

Francken, J. C., Gaal, S. van, \& de Lange, F. P. (2011). Immediate and long-term priming effects are independent of prime awareness. Consciousness and Cognition, 20(4), 1793-1800. https://doi.org/ 10.1016/j.concog.2011.04.005

Geyer, T., Baumgartner, F., Müller, H. J., \& Pollmann, S. (2012). Medial temporal lobe-dependent repetition suppression and enhancement due to implicit vs. explicit processing of individual repeated search displays. Frontiers in Human Neuroscience, 6, 272. https://doi.org/ 10.3389/fnhum.2012.00272

Griffin, I. C., Miniussi, C., \& Nobre, A. C. (2001). Orienting attention in time. Frontiers in Bioscience, 6, 660-671.

Hannula, D. E., \& Greene, A. J. (2012). The hippocampus reevaluated in unconscious learning and memory: at a tipping point? Frontiers in Human Neuroscience, 6, 80. https://doi.org/10.3389/fnhum.2012. 00080

Henke, K. (2010). A model for memory systems based on processing modes rather than consciousness. Nature Reviews. Neuroscience, 11(7), 523-532. https://doi.org/10.1038/nrn2850

Heuer, H., \& Schmidtke, V. (1996). Secondary-task effects on sequence learning. Psychological Research, 59(2), 119-133. https://doi.org/ 10.1007/BF01792433

Jaramillo, S., \& Zador, A. M. (2011). The auditory cortex mediates the perceptual effects of acoustic temporal expectation. Nature Neuroscience, 14(2), 246-251. https://doi.org/10.1038/nn.2688

Jepma, M., Wagenmakers, E. J., \& Nieuwenhuis, S. (2012). Temporal expectation and information processing: A model-based analysis. Cognition, 122(3), 426-441. https://doi.org/10.1016/j.cognition. 2011.11.014

Lange, K., \& Röder, B. (2006). Orienting attention to points in time improves stimulus processing both within and across modalities. Journal of Cognitive Neuroscience, 18(5), 715-729. https://doi. org/10.1162/jocn.2006.18.5.715

Mathewson, K. E., Fabiani, M., Gratton, G., Beck, D. M., \& Lleras, A. (2010). Rescuing stimuli from invisibility: Inducing a momentary release from visual masking with pre-target entrainment. Cognition, 115(1), 186-191. https://doi.org/10.1016/j.cognition.2009.11.010

Max, C., Widmann, A., Schröger, E., \& Sussman, E. (2015). Effects of explicit knowledge and predictability on auditory distraction and target performance. International Journal of Psychophysiology, 98(2), 174-181. https://doi.org/10.1016/j.ijpsycho.2015.09.006

Menceloglu, M., Grabowecky, M., \& Suzuki, S. (2017). Comparing the effects of implicit and explicit temporal expectation on choice response time and response conflict. Attention, Perception \& Psychophysics, 79(1), 169-179. https://doi.org/10.3758/s13414016-1230-4

Nissen, M. J., \& Bullemer, P. (1987). Attentional requirements of learning: Evidence from performance measures. Cognitive Psychology, 19(1), 1-32. https://doi.org/10.1016/0010-0285(87)90002-8

Nobre, A. C., \& Rohenkohl, G. (2014). Time for the Fourth Dimension in Attention. In A. C. Nobre \& S. Kastner (Eds.), The Oxford Handbook of Attention (pp. 676-724). Oxford University Press.

Preston, A. R., \& Gabrieli, J. D. E. (2008). Dissociation between Explicit Memory and Configural Memory in the Human Medial Temporal Lobe. Cerebral Cortex, 18(9), 2192-2207. https://doi.org/10.1093/ cercor/bhm 245

Ratcliff, R. (1979). Group reaction time distributions and an analysis of distribution statistics. Psychological Bulletin, 86(3), 446-461. Retrieved from http://www.ncbi.nlm.nih.gov/pubmed/451109

Ratcliff, R., \& McKoon, G. (2008). The Diffusion Decision Model: Theory and Data for Two-Choice Decision Tasks. Neural
Computation, 20(4), 873-922. https://doi.org/10.1162/neco.2008. 12-06-420

Ratcliff, R., \& Rouder, J. N. (1998). Modeling Response Times for TwoChoice Decisions. Psychological Science, 9(5), 347-356. https:// doi.org/10.1111/1467-9280.00067

Rohenkohl, G., Cravo, A. M., Wyart, V., \& Nobre, A. C. (2012). Temporal expectation improves the quality of sensory information. The Journal of Neuroscience : The Official Journal of the Society for Neuroscience, 32(24), 8424-8428. https://doi.org/10.1523/ JNEUROSCI.0804-12.2012

Sanchez, D. J., \& Reber, P. J. (2013). Explicit pre-training instruction does not improve implicit perceptual-motor sequence learning. Cognition, 126(3), 341-351. https://doi.org/10.1016/j.cognition. 2012.11.006

Sanders, A. F. (1975). The foreperiod effect revisited. The Quarterly Journal of Experimental Psychology, 27(4), 591-598.

Schlagbauer, B., Muller, H. J., Zehetleitner, M., \& Geyer, T. (2012). Awareness in contextual cueing of visual search as measured with concurrent access- and phenomenal-consciousness tasks. Journal of Vision, 12(11), 25-25. https://doi.org/10.1167/12.11.25

Schroeder, S. C. Y., Ball, F., \& Busch, N. A. (2018). The role of alpha oscillations in distractor inhibition during memory retention. European Journal of Neuroscience. https://doi.org/10.1111/ejn. 13852

Seitz, A. R. (2017). Perceptual learning. Current Biology, 27(13), R631R636. https://doi.org/10.1016/j.cub.2017.05.053

Seitz, A. R., \& Watanabe, T. (2009). The phenomenon of task-irrelevant perceptual learning. Vision Research, 49(21), 2604-2610. https:// doi.org/10.1016/j.visres.2009.08.003

Shen, D., \& Alain, C. (2012). Implicit temporal expectation attenuates auditory attentional blink. PloS One, 7(4), e36031. https://doi.org/ 10.1371/journal.pone.0036031

Stefaniak, N., Willems, S., Adam, S., \& Meulemans, T. (2008). What is the impact of the explicit knowledge of sequence regularities on both deterministic and probabilistic serial reaction time task performance? Memory \& Cognition, 36(7), 1283-1298. https://doi.org/10. 3758/MC.36.7.1283

Summerfield, C., \& Egner, T. (2009). Expectation (and attention) in visual cognition. Trends in Cognitive Sciences, 13(9), 403-409. https://doi.org/10.1016/j.tics.2009.06.003

Turk-Browne, N. B., Scholl, B. J., Chun, M. M., \& Johnson, M. K. (2009). Neural Evidence of Statistical Learning: Efficient Detection of Visual Regularities Without Awareness. Journal of Cognitive Neuroscience, 21(10), 1934-1945. https://doi.org/10. 1162/jocn.2009.21131

Turk-Browne, N. B., Scholl, B. J., Johnson, M. K., \& Chun, M. M. (2010). Implicit perceptual anticipation triggered by statistical learning. The Journal of Neuroscience : The Official Journal of the Society for Neuroscience, 30(33), 11177-11187. https://doi.org/10. 1523/JNEUROSCI.0858-10.2010

Van den Bussche, E., Vermeiren, A., Desender, K., Gevers, W., Hughes, G., Verguts, T., \& Reynvoet, B. (2013). Disentangling conscious and unconscious processing: a subjective trial-based assessment approach. Frontiers in Human Neuroscience, 7, 769. https://doi.org/ 10.3389/fnhum.2013.00769

Vandekerckhove, J., \& Tuerlinckx, F. (2007). Fitting the Ratcliff diffusion model to experimental data. Psychonomic Bulletin \& Review, 14(6), 1011-1026. Retrieved from http://www.ncbi.nlm.nih.gov/pubmed/ 18229471

Vandekerckhove, J., \& Tuerlinckx, F. (2008). Diffusion model analysis with MATLAB: a DMAT primer. Behavior Research Methods, 40(1), 61-72. Retrieved from http://www.ncbi.nlm.nih.gov/ pubmed/18411528

Visser, T. A. W., Ohan, J. L., \& Enns, J. T. (2015). Temporal cues derived from statistical patterns can overcome resource limitations in the 
attentional blink. Attention, Perception \& Psychophysics, 77(5), 1585-1595. https://doi.org/10.3758/s13414-015-0880-y

Visser, T. A. W., Tang, M. F., Badcock, D. R., \& Enns, J. T. (2014). Temporal cues and the attentional blink: a further examination of the role of expectancy in sequential object perception. Attention, Perception \& Psychophysics, 76(8), 2212-2220. https://doi.org/10. 3758/s13414-014-0710-7

Wagenmakers, E.-J., Love, J., Marsman, M., Jamil, T., Ly, A., Verhagen, J., .. Morey, R. D. (2018). Bayesian inference for psychology. Part II: Example applications with JASP. Psychonomic Bulletin \& Review, 25(1), 58-76. https://doi.org/10.3758/s13423-017-1323-7

Wang, P., \& Nikolić, D. (2011). An LCD Monitor with Sufficiently Precise Timing for Research in Vision. Frontiers in Human Neuroscience, 5(85), 85. https://doi.org/10.3389/fnhum.2011.00085
Westerberg, C. E., Miller, B. B., Reber, P. J., Cohen, N. J., \& Paller, K. A. (2011). Neural correlates of contextual cueing are modulated by explicit learning. Neuropsychologia, 49(12), 3439-3447. https:// doi.org/10.1016/j.neuropsychologia.2011.08.019

Zhang, J., \& Rowe, J. B. (2014). Dissociable mechanisms of speedaccuracy tradeoff during visual perceptual learning are revealed by a hierarchical drift-diffusion model. Frontiers in Neuroscience, 8 , 69. https://doi.org/10.3389/fnins.2014.00069

Publisher's note Springer Nature remains neutral with regard to jurisdictional claims in published maps and institutional affiliations. 